International Journal of Wireless \& Mobile Networks (IJWMN) Vol. 3, No. 3, June 2011

\title{
Why the Accuracy of the Received Signal Strengths as a Positioning Technique was not accurate?
}

\author{
Samir A. Elsagheer Mohamed \\ Electrical Engineering Department, Faculty of Engineering, South Valley University, Aswan, Egypt. \\ In sabbatical leave in College of Computer, Qassim University, P.O.B 6688, Buryadah 51453, KSA. \\ Emails: samhmd@qu.edu.sa; samirahmed@yahoo.com
}

\begin{abstract}
There is a great need for having reliable and accurate positioning technique for many wireless network technologies such as the Wireless Ad Hoc Networks, Wireless Sensor Networks, Vehicular Ad Hoc Networks, and Wireless Local Area Networks. In addition, the Mobile Computing which still in the development phase requires good localization system for the mobile nodes. The system must allow each node to know precisely its current position or location in real time. Despite the many works that exist in the literature in this area, all of the proposals have some limitations. The features of optimal system are accurate, reliable, scalable, lightweight, and inexpensive. There are many works for the use of the Received Signal Strength (RSS) as a positioning system. However, in these works the accuracy of the positioning is very bad, about 50 meters. In this paper, we provide positioning technique based on the RSS and satisfy the mentioned optimal system features. The obtained experimental results showed an accuracy that can be better than only 2 meters. This huge improvement is due to the way by which the system is used to minimize the radio interference and the near-far problems.
\end{abstract}

\section{KEYWORDS}

Positioning Systems; Localization Systems; Neural Networks; Wireless Networks; Received Signal Stength; Curve Fitting.

\section{INTRODUCTION}

With the advance in the wireless networks technologies and their vast deployment, the demand on having precise localization or positioning system increases. Such system allows any wireless node (mobile device) to know its position in real time. The technologies that require such system are Wireless Local Area Networks [1], Wireless Ad-hoc Networks [2], Mobile Ad-hoc Networks [3], Wireless Sensor Networks [4], and Vehicular Ad-hoc Networks (VANET) [5-8]. Another new technology that requires a localization system is the Mobile Computing [9].

There exist several positioning techniques that have been proposed for that matter. However, the limitations in many of them make them unfeasible in practice on in wide deployment. To further detail this point, we have to know the features of the optimal system, which are accurate, reliable, scalable, lightweight, and inexpensive. No existing system can satisfy all these features at once. Lack of accuracy of the resulting measurements is the most unacceptable disadvantage. The most common positioning system in the world is the GPS [10][11] (a GPS receiver calculates its global position from the signals received from at least three satellites that feeds information about their positions). GPS has the following features: not accurate, not reliable, scalable, complex, and expensive. GPS devices can produce an error of up to 50 meters [10]. Recently an enhancement to the GPS referred as the Differential GPS (DGPS) consisting in installing expensive ground stations can improve the accuracy significantly. However, GPS and DGPS and the similar techniques do not work in tunnels, undergrounds, and in high dense 
building areas, because the signal cannot be received or it is received very weak. This limits the use of GPS and hence DGPS in many applications (e.g. the Sensor Networks and indoor applications). Other positioning techniques are provided in the Related Works Section.

A survey in the literature reveals that the use of the Received Signal Strength (RSS) [1][12][13][14] as a positioning technique can satisfy all the optimal features except the most important one: the accuracy. Wireless networks operate by emitting radio signals that propagate in the surroundings of the transmitter. The Signal Strength decays with the distance from the emitter. Thus, any node in the covering area of the emitter can estimate the distance to that emitter based on the Received Signal Strength (RSS). The nice features of this technique are the following. No extra hardware is required, thus it is inexpensive or free. It is scalable, as any node in the network can use it as the signals are broadcasted by the emitter and hence received by all the nodes in the covering area. It is know that the wireless networks use broadcast channels. It is reliable given that the wireless nodes are located in the covering area of the emitters. Finally, it is not complex, as simple calculation can reveal an estimate of the relative position of the node based on the RSSs. Regarding the accuracy of the RSS techniques, the existing works have one common issue: lack of accuracy (sometimes more than 50 meters as accuracy [12]).

In this Paper, we present a new methodology by which the RSS technique can provide the optimal system features. The main advantage of the proposed technique is about the accuracy. Huge improvement of the accuracy is obtained and verified by the experimental measurements. This is mainly to the way by which the radio sources are configured. That led to solve two main drawbacks: the interference and the near-far problems commonly found in the wireless networks. By minimizing the interference, the RSS gives better estimate of the distance. Furthermore, by not using the radio source signals that are very strong (when the wireless node is near to the radio source), the RSS gives a true estimate of the distance. By solving these two problems, a significant accuracy improvement is obtained. The proposed technique is very lightweight. We propose two systems: one that uses the Neural Networks (NN) and the second that uses a simple curve fitting which gives a maximum error of only 2 meters.

The rest of this paper is organized as follows: In Section 2, the related works and research efforts are given. Experimentations and data analysis that led to the discovery for improving the accuracy using RSS techniques are given in Section 3. Descriptions of the proposed techniques are given in Section 4. Experimentations and the obtained results are given in Section 5. Finally, the Conclusions and the future works are given in Section 6.

\section{RELATED WORKS}

There exits two categories of positioning systems: indoors techniques [16][24][25][26] and outdoor techniques. We focus here on the outdoor techniques. Existing outdoor positioning techniques include the followings. The Time-Of-Arrival (TOA) [11] based techniques which are based on the travelled distance from the base station to the receiver of a known signal. TOA techniques require a perfect synchronization between the clocks of the base stations and the receivers. This cannot be guaranteed except by using atomic clocks which are very expensive. Otherwise the accuracy is compromised. The Direction-of-Arrival (DOA) or Angle-of-Arrival (AOA) techniques require multidirectional antennas and complex hardware [11]. A composed technique referred as TOA-DOA is used in [3]. Finally, there exists techniques that use the round trip time [11]. The receiver sends a small packet to the base station and wait for a reply from it. The elapsed time is proportional to the distance between it and the base station. By some calculations the receiver can know its location provided that it can talk to at least three base stations which know their accurate locations. Again the distance between the base stations and the receiver must be large to obtain good results. 
The most famous one which is always mentioned in the research papers is the GPS [10][11]. However, GPS has several drawbacks as discussed below:

- Its accuracy, the civilian GPS has a limited accuracy in the order of 50 meters in each direction. A survey of the most famous GPS device vendors, the best announced accuracy is $+/-5$ meters, which gives an error of about 10 meters in each direction. They say that this accuracy is obtained in $95 \%$ of all the readings. The other $5 \%$ of the readings, the accuracy may be very bad. This still too much far away of being acceptable in the critical applications requiring a precise positioning system.

- In some places (e.g. inside tunnels, undergrounds, etc.), the GPS has no coverage. This is because the GPS receivers calculate the position based on the signals received from 4 simultaneous satellites. In such places, the received signals are too weak or no signals can be received at all. Therefore, the GPS receivers cannot calculate the position.

- GPS signals sent from the satellites can be simulated. In other words, there exist GPS simulators (devices generating fake GPS signals). As mentioned before, the real GPS signals are relatively weak signals. GPS simulators produce relatively strong signals and therefore, the GPS receiver will use the fake signals and consider the real signals background noise. As a result, the receiver will calculate the position based on the fake signals. Thus, any jammer equipped by this kind of GPS simulator can inject erroneous data that leads to make the vehicle believe that it is in completely different position.

Depending on the wireless network technology, there are several studies proposing several poisoning techniques. Examples are: Wireless Local Area Networks [1][27], Wireless Ad-hoc Networks [2], Mobile Ad-hoc Networks [3], Wireless Sensor Networks [4], Vehicular Ad-hoc Networks (VANET) [11] and Mobile Computing [9].

\section{FIELD EXPERIMENTS TO MEASURE RSSS FROM WAPS.}

In order to develop and validate the positioning system based on the RSS, two field experiments are conducted in realistic conditions. In wireless network configurations, the IEEE 802.11g standard is used. The network architecture consists of having Wireless Access Points (WAPs) installed in fixed and know locations. A Mobile Unit (MU) which is simply a Laptop having the software that can measure the RSS from the WAPs at any place in the covering area is used. In both experiments, the WAPs are installed in a straight line and the distance between each two successive WAPs is $100 \mathrm{~m}$. The MU moves in a line parallel to the WAPs line and it measures the RSSs from all the WAPs at predefined location. We have used these field experiments' data for a positioning technique for the Vehicular Ad-Hoc Networks [15].

\subsection{The first experiment}

The environment and the precautions that we take for this experiment are as follows. The MU measures the RSSs each $5 \mathrm{~m}$ from the starting point (location $0 \mathrm{~m}$ ) until the ending point (location $200 \mathrm{~m}$ ). The elevation of the access points is $110 \mathrm{~cm}$, above the ground. Distance between the line of access points and the parallel line of the test line equals $7 \mathrm{~m}$. All the access points and mobile host operates on Channel 6. A diagram of the setup of this experiment is shown in Figure 1. 


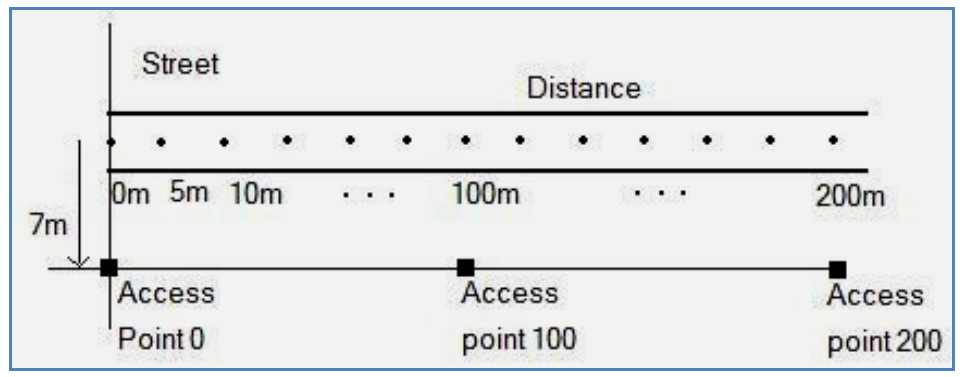

Figure 1. Experiment \#1 Setup

\subsubsection{Obtained Results for Experiment \#1}

In this Section, we will present the obtained results. In all the Figures in this Section, the $\mathrm{x}$ axis shows the distance starting from the $0 \mathrm{~m}$ location. The $y$-axis shows the value of the received signal strength intensity (RSS) from the wireless access point(s) WAPs. Remember, the RSS is negative and the far we are from the WAP, the less the RSS.

Figure 2 shows the obtained RSS values from the three Access Points for the First Experiment (Experiment \#1). From the shown data, we can see that the results are very bad, the data is very chaotic. It is expected that the RSS from any WAP decrease when we move far away of it (increasing the distance). However, for all the WAPs, this is not the case. By inspecting the results, we can expect that no way to obtain accurate estimate of the position based on the RSSs. On other words, the accuracy will be very bad. That expectation is confirmed using the neural network as a learning tool (See Section 4.1.2). The accuracy is very bad, about 80 meters in any direction (for the lack of space, results are omitted).

A very interesting finding here is that this obtained result by the neural network $(\mathrm{NN})$ is almost the same as those found in the literature [12][11]. It seems that the existing work in the RSS techniques do not take into consideration the effect of the channel interference.

\subsubsection{Why the results are chaotic?}

That was the first experiment that we carried out. Once we collected the results, we have thought in the problem. We, then, realized that all the WAPs operated on the same frequency channel. It is known that the Wi-Fi networks have 11-13 channels depending on the country. If all the WAPs use the same channel, that will cause significant noise and interference. Another problem is that this experiment setup suffers from the near-far problem described before. 


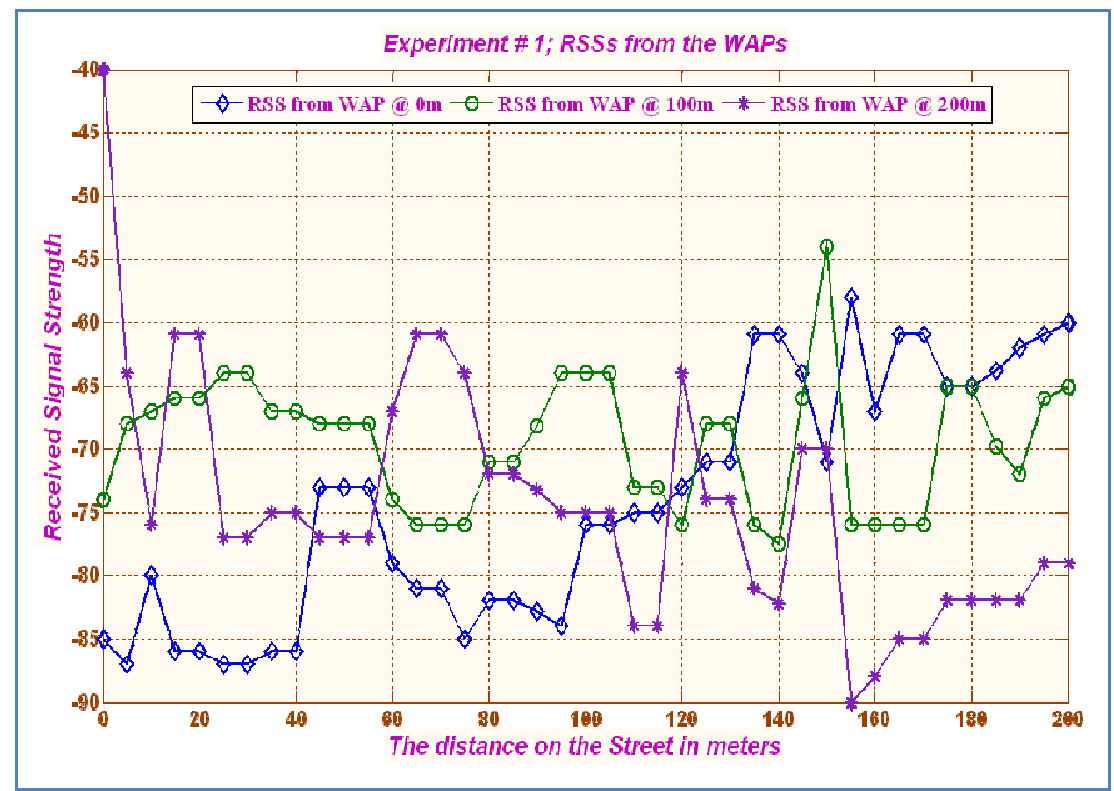

Figure 2. Obtained RSS from the three Access Points for the First Experiment (Experiment $\# 1)$

\subsection{The Second Field Experiment}

In this experiment our goal is to solve a fatal problem discovered in Experiment \#1. It is known that the channels in IEEE 802.11 standards do not overlap if they are more than 5 channels apart. For example, if one WAP use channel \#1, the second uses channel \#6 and the third uses channel \#11, then there is not overlap and thus no noise nor interference that can degrade the results. That is confirmed using the obtained results. Thus in this experiment, to reduce the radio channel interference and hence increase the accuracy of the RSSI, each WAP operates on a non-overlapping channel (Chanel \#1, 7, and 13). All other experiment setup is the same as the previous one. We have repeated the experiment three times with different configurations. We obtained the same results in each case. Thus, in this Section, only one of the results will be presented and analyzed.

\subsubsection{Experiment \#2 obtained results}

Figure 3 depicts the obtained RSS values from the three Access Points for Experiment \#2. 


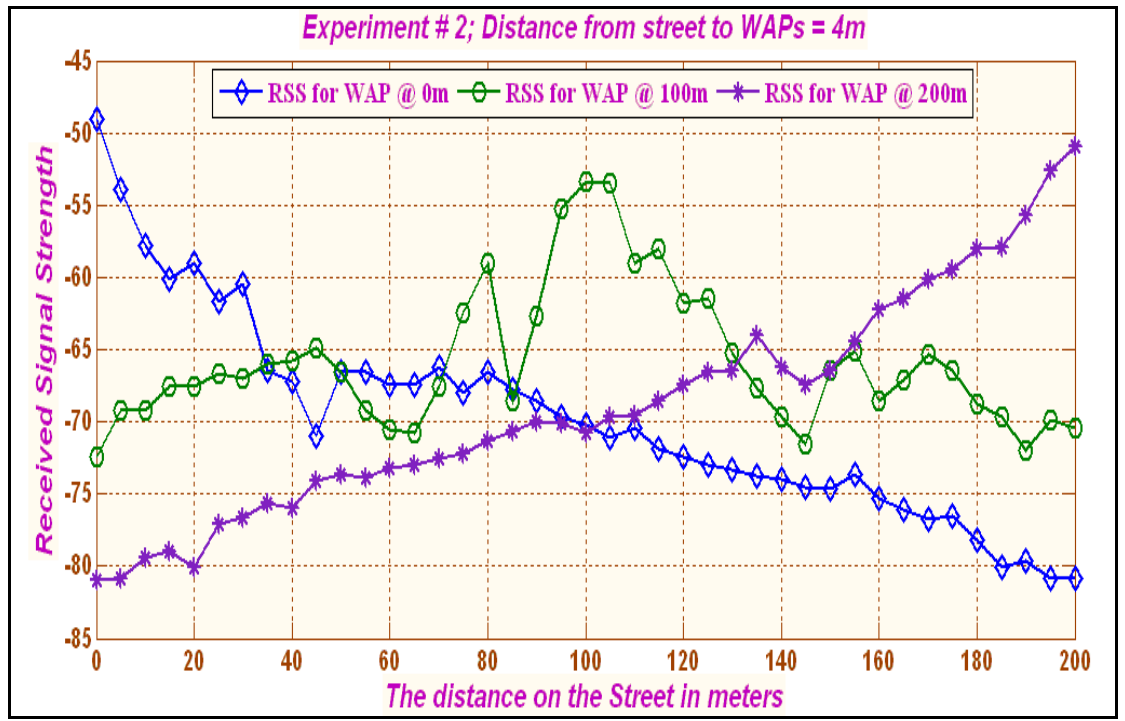

Figure 3. Obtained RSS from the three Access Points for the Second Experiment (Experiment \#2).

\subsubsection{Data Analysis of Experiment \#2}

As can be seen from Figure 3, once the interference of the channels is eliminated, the results are improved significantly. Moreover, one of the most important discoveries in this work is that the RSS from any WAP is still chaotic even if there is no interference if the distance to the WAP is less than 60 meters. When the MU moves away from the WAP, we obtain better results (better estimate of the distance based on the RSS). This can be seen for WAP@0m and WAP@200m. When the distance is greater than 60 meters, the RSS is inversely proportional to the distance (the expected behavior). For WAP@100m, this is also true. In the middle part of Figure 3, the data are chaotic. However, it starts to be correct at the boundaries.

In the next Sections we present the use of the neural networks and the curve fitting to estimate the position of the vehicle based on the received signal strengths and the use of the obtained experimental data.

\section{Two Competitive Positioning Systems Using RSS}

Based on the results of the presented field experiments and the data analysis, two positioning systems are presented in this Section. First, we explore the use of the Neural Networks and we analysis their performance and limitations. Second, a simple, but efficient technique using the curve fitting is proposed.

\subsection{A positioning system using the Neural Networks}

The use of the neural networks (NNs) as a learning tool to estimate the location of the MU based on the RSS from the WAPs is presented in this Section. The full theory and the description of the $\mathrm{NN}$ are beyond the scope of this paper. However, the basic functionality of the neural networks is given here. The idea of the NN is inspired from the biological nervosystem. Similarly to the biological neuron, the artificial neural network learns by experience. In other words, a set of learning examples must be collected for the problem in hand. These samples are simply a set of the system inputs and their corresponding outputs. Using training algorithms, the NN will learn the system behavior based on these examples. Once trained, the neural network will act as the system. For any new inputs, the $\mathrm{NN}$ will estimate the outputs based on the learning experience. 


\subsubsection{Description of the approach with the NN}

Matlab 2009a is used to do most of the calculations, and the Figures in this paper. Especially, the powerful toolbox in Matlab called the 'Neural Network toolbox' is used for all the work in this Section.

The inputs to the NN are the RSS from the WAPs. The output is the location of the MU in the field. The NN needs to be trained, validated then tested. Thus, using Matlab, the whole learning example (the set of samples in the form RSS-Position pairs), is divided into three sets: one for training; the second for validation; and the third for testing. This is done automatically by Matlab. The ratios are $70 \%$ for training; $15 \%$ for validation; and $15 \%$ for testing. The training stops when the performance on the validation set increase. This is to avoid a known problem with the NN which is the overtraining.

For the NN, we use the Feedforward architecture that contains the input layer connected to the inputs; the output layer, which gives the estimate of the location based on the learning experience. In addition, there is the hidden layer. This layer can contain a given number of hidden neurons. Moreover, the random seed for the random generator which is used to assign the initial weights of the NN is very important. For the same samples, and the same NN, but different random seeds, the trained networks are completely different. Thus, we take this as a parameter for all the data.

A Matlab script to automate most of the work is developed. Then the outputs can be saved and restored at any time to know the best $\mathrm{NN}$ that gives the best performance.

\subsubsection{Working with data obtained in experiment \#1}

We have run the script on the data obtained from experiment \#1. A total of 56 different NN architectures are created; trained; tested and validated. The performance measures are also calculated on both the testing and the whole samples.

Figure 4 shows the difference between the actual data (distance) and those estimated by the trained $\mathrm{NN}$ for the best one. As we can see from the Figure, error on the positioning may reach up to $60 \mathrm{~m}$. This is very high and not suitable for most the wireless networks. This bad result is that found on the literature, see for example [11]. Actually, people used the technique without taking into account the effect of the interference problem resulting from using the same channel for all the WAPs. This is why the published results for the mechanisms using the RSSs are not encouraging. 


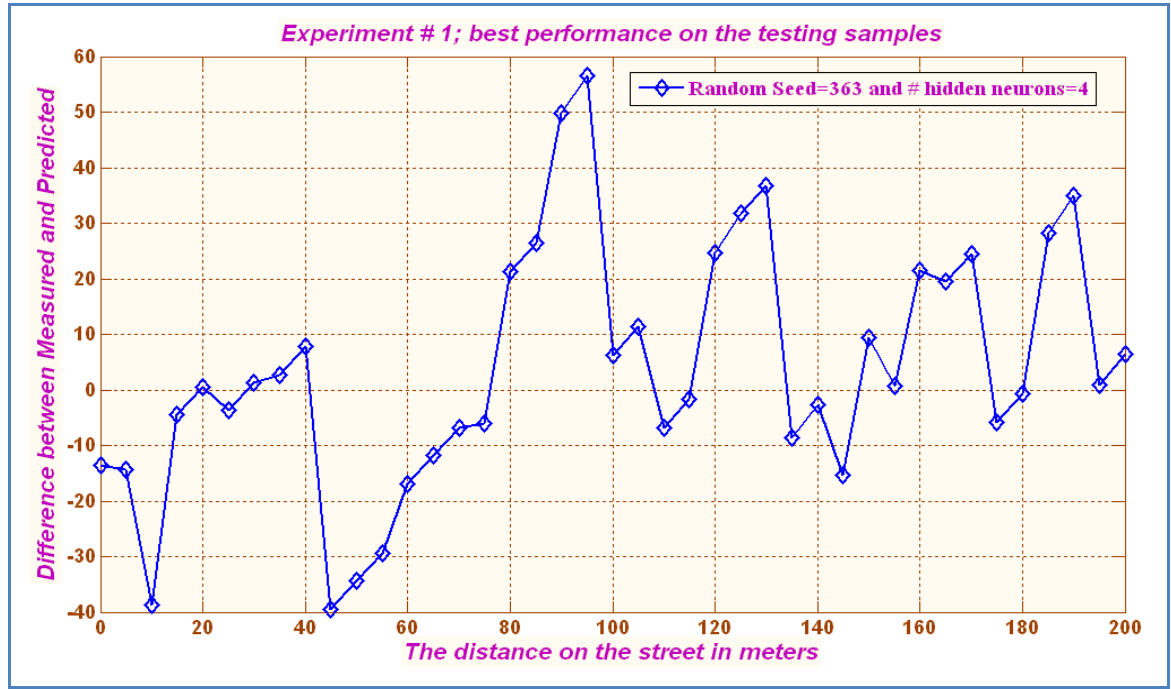

Figure 4. The performance of the best NN for experiment \#1. The Figure shows the difference between the measured data experimentally and those calculated by the NN. The error can reach up to 60 meters. Correlation coefficients $=93 \%$

\subsubsection{Working with data of experiment \#2}

Similar to what done in experiment \#1, the whole work is repeated on the data for experiment \#2. We have run the script on the data obtained from experiment \#2. By using NNs with different hidden neurons and random seed generator: a total of 180 different NNs are trained, validated and tested. The performance measures are also calculated on both the testing and the whole samples. In Table 1, we show the obtained data sorted by the MSE on the whole samples and the max error obtained. In the table, we show also the standard deviation, the max error, the variance and the correlation for both the testing samples and the whole samples.

From this table, we can see that the best NN is obtained when the \# of hidden neurons is 10 and for initial random seed $=102$. As expected the accuracy of this best one is very good compared to that of the first experiment. The MSE of the best NN is 6 against 172 for the first experiment. The standard deviation is about $3.2 \mathrm{~m}$, the max error is about 7.6 meters against 60 meters. Comparing these results to the GPS, we can see that we can reach accuracy better than the GPS. Thus, our technique can be used to complement the GPS in the areas where the GPS signal cannot be received or if it is very week.

Thus, the accuracy is improved significantly, by about a factor of 7 . That is a huge improvement. This can also be seen from Figure 5 which depicts the difference between the actual data (distance) and those estimated by the trained $\mathrm{NN}$.

\subsubsection{Effect of removing the WAP@100m}

We have removed the RSS from WAP@100m that we have thought may degrade the performance. The results show no further improvement (omitted for the sake of space). This is because three WAPs are better than two. 
International Journal of Wireless \& Mobile Networks (IJWMN) Vol. 3, No. 3, June 2011

Table 1. Results of exp. \#2. By using NNs with different hidden neurons and random seed generator: A total of 180 different NNs are trained, validated and tested; only the top 5 are shown

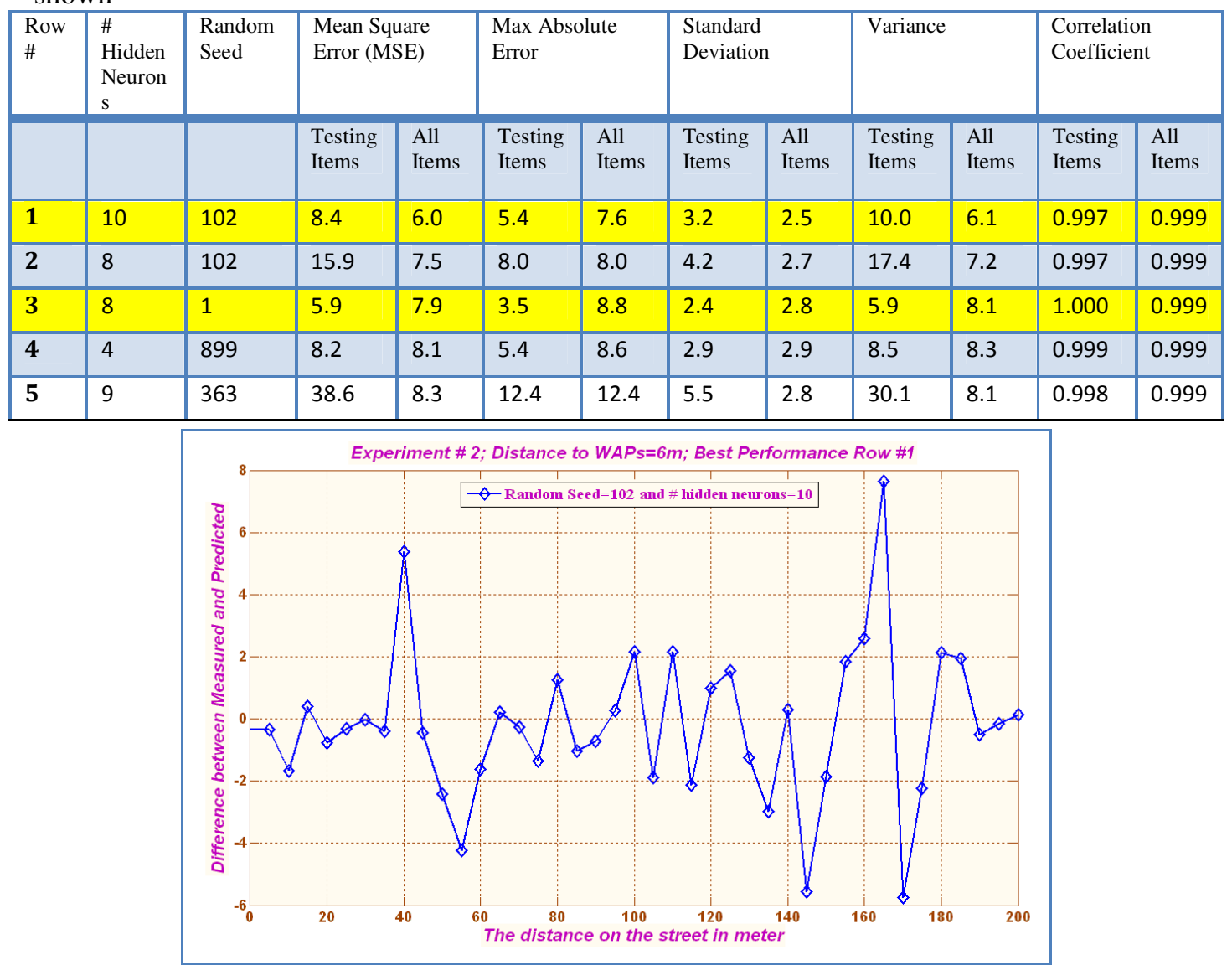

Figure 5. The performance of the best $\mathrm{NN}$ for experiment \#2. The Figure shows the difference between the measured data experimentally and those calculated by the NN.

\subsubsection{Using only one WAP but far away}

We are seeking to improve the performance as much as it possible. Thus, in this subsection, we will use data from WAP@0m and WAP@200m only when the distance from each one is greater than $60 \mathrm{~m}$. This is to eliminate the source of error due to the chaotic part of the curve (near-far problem). The obtained results also are not encouraging. Again, the NN cannot correctly learn from the few samples available for training. The next subsection presents another way to improve the performance, which is the curve fitting.

\subsection{Positioning System Using Curve Fitting}

In the previous Section, we have seen how to use the neural network for the positioning system. However, it is still in the range of 7 meters. We are seeking to improve this performance. We have explored the use of the curve fitting to obtain a polynomial equation that can be used to obtain the distance from the WAP given its RSS. Several type of curve fittings are evaluated using Matlab. The best results is given bellow. We aim to approximate the positioning problem according to the following equation. For the Linear model Polynomial of degree 4, we have the following equation:

$$
\text { Distance }=p_{1} R^{4}+p_{2} R^{3}+p_{3} R^{2}+p_{4} R+p_{5},
$$


where $R$ is the received signal strength.

\subsubsection{Starting from $60 \mathrm{~m}$}

We have used the data from WAP@200m and by removing the data when the distance is less than $60 \mathrm{~m}$ (the chaotic data). Using Matlab "cftool" curve fitting tool, the estimated values of the Coefficients (with $95 \%$ confidence bounds) are as follows:

$p_{1}=-0.005206, p_{2}=-1.553, p_{3}=-173.5, p_{4}=-8608, p_{5}=-1.601 \mathrm{e}+005$.

The obtained Goodness of fit for the above data is: SSE: 422.7; R-square: 0.9917; Adjusted R-square: 0.9903; and RMSE: 4.197. The curve fitting results are shown in Figure 6. We have calculated the distance for the given RSSs in the range. In Figure 7, we draw the difference between the actual data (measured) and the calculated ones. One can see that using only one WAP, the accuracy is almost the same as using the NN with three WAP. That is a very important finding.

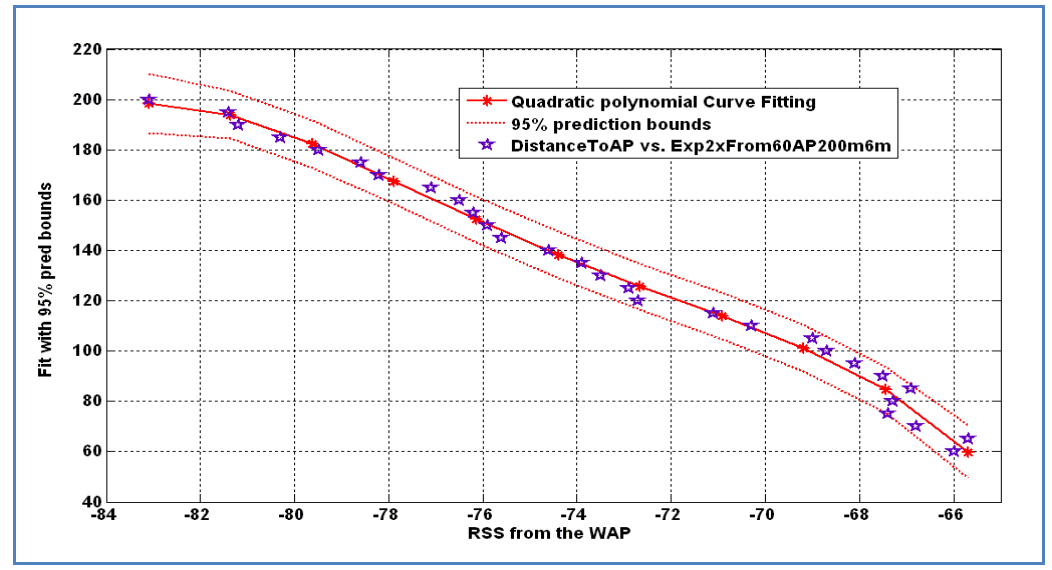

Figure 6. Curve Fitting results using cftool, when the Distance is greater than $60 \mathrm{~m}$

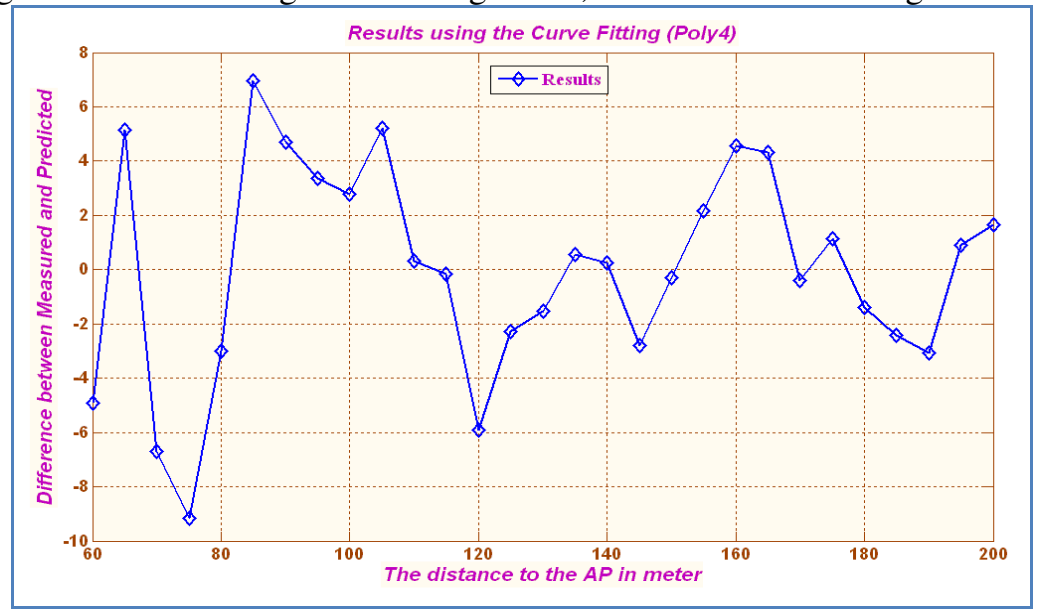

Figure 7 The performance curve fitting for Exp \# by using only data from WAP@ 200m and excluding data before $60 \mathrm{~m}$. The Figure shows the difference between the measured data experimentally and those calculated by the $\mathrm{NN}$.

\subsubsection{Starting from $100 \mathrm{~m}$ (solving the near-far problem)}

From the previous section, we can see that the performance is bad at the beginning of the curve. However, it is getting better when the distance from the WAP increase. Thus, we redid the 
same experiment, but removing all the data when the distance is less than $100 \mathrm{~m}$. The results of the curve fitting are shown in Figure 8.

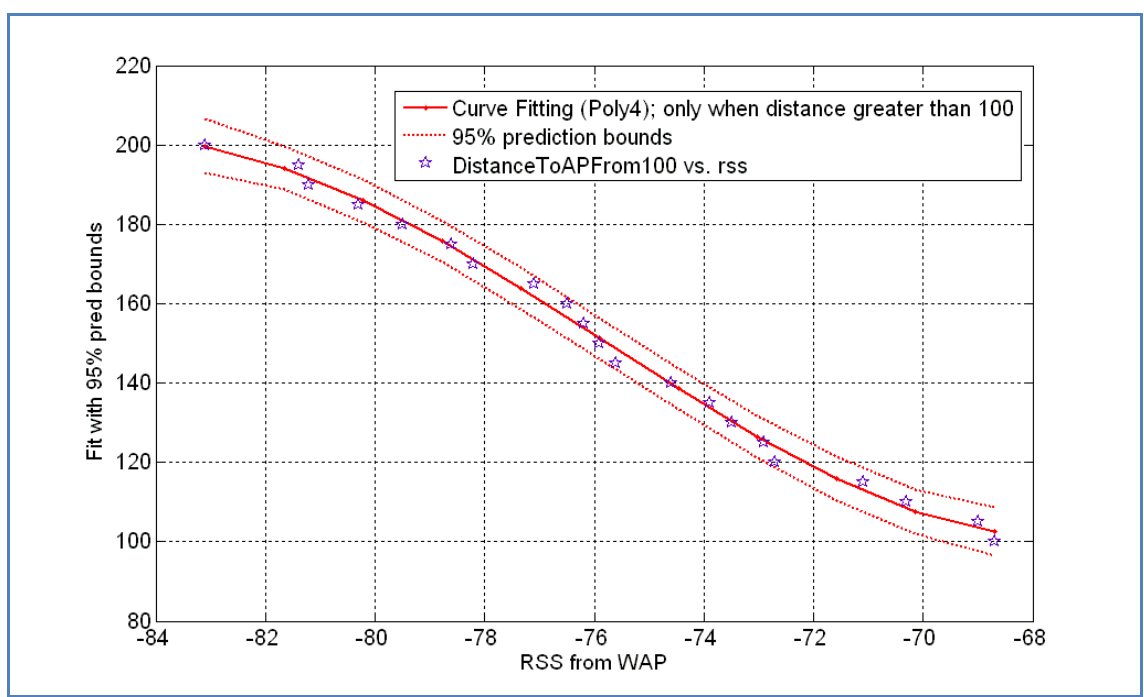

Figure 8. Curve Fitting results using cftool, when the Distance is greater than 100m

The coefficients of the polynomial equation are as follows: $p_{1}=0.0004373, p_{2}=0.1736, p_{3}=24.4$, $p_{4}=1458, p_{5}=3.171 \mathrm{e}+004$. The obtained goodness of fit is: $\mathrm{SSE}=85.13$, R-square $=0.9956$, Adjusted R-square $=0.9945, \mathrm{RMSE}=2.307$. Comparing these data with the ones in the previous experiment, we note a very significant improvement. This is also confirmed from Figure 9. We can see that the accuracy is improved (the max error is now 4 meters instead of 8 meters). This is by using only one WAP. Again, if we inspect the figure carefully, we can see that the results when the distance is greater than $170 \mathrm{~m}$ are within only 2 meters (A very accurate results). This result is much better that that obtained using the traditional GPS.

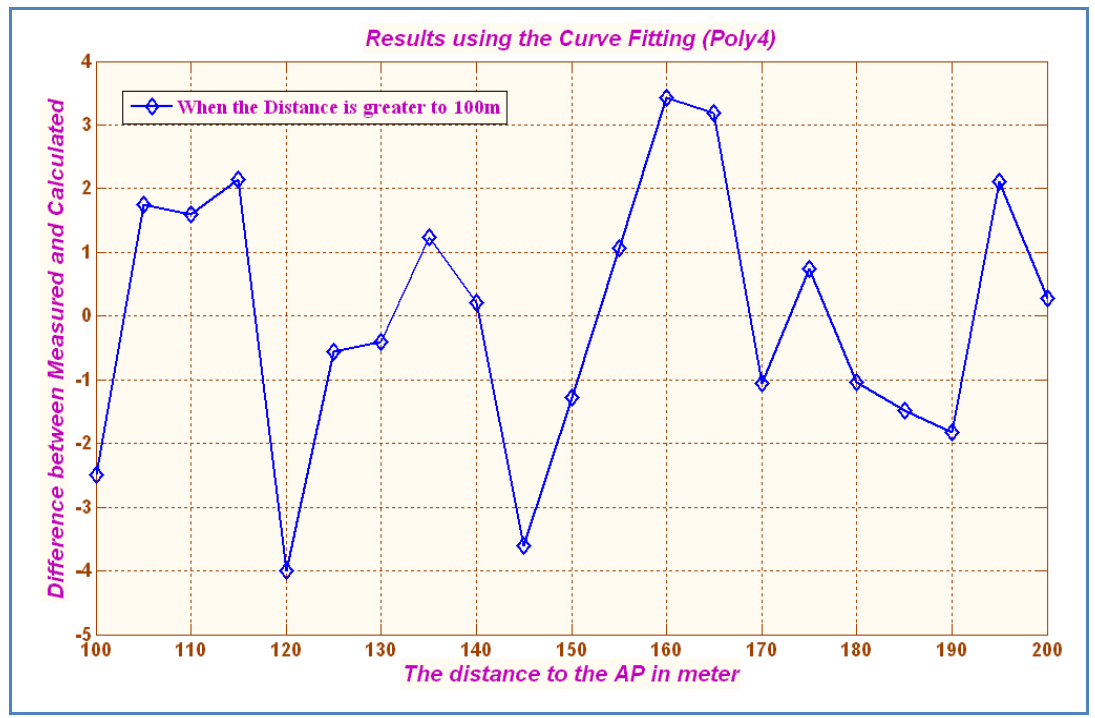

Figure 9. The performance curve fitting for Exp \#2 using only data from WAP@ 200m and excluding data before $100 \mathrm{~m}$. The Figure shows the difference between the measured data experimentally and those calculated by the NN. 
International Journal of Wireless \& Mobile Networks (IJWMN) Vol. 3, No. 3, June 2011

\section{THE BEST APPROACH FOR THE POSITIONING SYSTEM}

Based on all the results explained in the previous Sections, we summarize here the best approach that can be used to build a system for an accurate positioning system using the RSSs. The resultant system gives accurate positioning result because the interference and the near-far problems are solved. In the wireless network geographical area, there must be several radio sources. For example, for the WLAN the wireless access points (WAPs) are the radio sources that periodically emit beacons. Second example, in the WSN, several reference WAPs have to be installed. Third example, in VANET, the Road-Side Unites will act as the WAP.

- The WAPs must be installed with, if possible, a minimum distance between each two of $200 \mathrm{~m}$. They have to cover the whole geographical area.

- At any point, the mobile unite (MU) unit must receive from at least two WAPs satisfying the next condition: these WAPs must be as far as possible (the less the RSS), and each operates on a different radio channel.

- In order to be able to obtain the position of the MU, it must hear from at least two WAPs, if the $2 \mathrm{D}$ location of the MU is desired. In the case of $3 \mathrm{D}$ positioning, at least three access points must be heard.

- If the MU hears from many WAPs, this will be very good. This can improve dramatically the accuracy. For example, in order to obtain the 2D position of the MU, only two WAPs are need. But if it receives from three, we find three readings. By taking the average of the three values, the accuracy will be improved. Adding one WAP can improve the $3 \mathrm{D}$ case very much.

- Using a simple triangulation technique, the position coordinate point of the MU can be determined.

- Each WAP knows its absolute location. Thus, the MU can determine its absolute location based on the estimated relative position and the absolute locations of the surrounding WAPs.

\section{The CONCLUSIONS AND FUTURE DIRECTIONS}

In this paper, an accurate generic positioning technique for the wireless networks is proposed. It is based on the Received Signal Strength (RSS). Although, the use of the RSS is not new, the way by which it is used in this paper to significantly improve the accuracy is new. The improvement of accuracy is due to tackling the problems of the channel interference and the near-far problems. The obtained results are an accuracy of 2 meters compared to 50 meters in most of the published works.

Field experiments are conducted on realistic situations. In the first one, the WAPs operate on the same channel. Hence, the interference caused degradation of estimating the distance from the RSS. In the second experiment, the results are very good compared to the first as the WAPs operated on different channels to reduce the interference. After analyzing the obtained results, we have proposed the use of the neural networks $(\mathrm{NN})$ to learn the positioning problem from the obtained learning examples. By using the curve fitting technique, and using only one WAP, we have done two experiments that show an obtained accuracy that can reach a maximum absolute error of only 2 meters. This can be obtained when the WAP is very far from the mobile unit. Future research directions include the exploration of the technique in the specific wireless technologies like the Wireless Sensor Networks. 


\section{REFERENCES}

[1] C. Komar, and C. Ersoy, "Location Tracking and Location Based Service Using IEEE 802.11 WLAN Infrastructure," in Proc. of the European Wireless Workshop, February 2004.

[2] Misra, S.; Guoliang Xue; Bhardwaj, S.; Secure and Robust Localization in a Wireless Ad Hoc Environment. IEEE Transactions on Vehicular Technology, Volume: 58, Issue: 3 2009, pp: 1480 1489.

[3] Zhonghai Wang and Zekavat, S.A., "A new TOA-DOA node localization for mobile ad-hoc networks: Achieving high performance and low complexity" IEEE 17th International Conference on Telecommunications (ICT), 2010, pp. $836-842$

[4] Weng Kai and Chen Chun; "Using RSS with difference method in localization algorithm for sensor networks", 2nd International Conference on Information Science and Engineering (ICISE), 2010; pp. $2500-2502$

[5] K. Saha and D. B. Johnson, "Modeling mobility for vehicular ad hoc networks," in Proc. of the ACM Workshop on Vehicular Ad hoc Networks (VANET), October 2004, pp. 91-92.

[6] R. Tatchikou, and S. Biswas, "Vehicle-to-vehicle packet forwarding protocols for cooperative collision avoidance", in Proc. of Globecom 2005, St. Louis, USA, Nov. 2005.

[7] A.Nandan, S. Das, G. Pau, M.Y. Sanadidi, and M. Gerla, "Cooperative downloading in Vehicular Ad Hoc Networks", in Proc. of WONS 2005, St. Moritz, Switzerland, Jan. 2005.

[8] S. Kato, S. Tsugawa, K. Tokuda, T. Matsui, and H. Fujii, "Vehicle control algorithms for cooperative driving with automated vehicles and intervehicle communications," IEEE Trans. on Intelligent Transportation Systems, vol. 3, no. 3, pp. 155-161, September 2002.

[9] Vaughan-Nichols, S.J. Will Mobile Computing's Future Be Location, Location, Location? Computer Journal, Volume: 42 , Issue: 2, DOI: 10.1109/MC.2009.65, 2009 , pp. 14 - 17

[10] Yu, K.; Guo, Y.J.; Anchor Global Position Accuracy Enhancement Based on Data Fusion. IEEE Transactions on Vehicular Technology, Vol.: 58, Issue: 3, 2009, pp.: 1616 - 1623

[11] M. Porretta, P. Nepa, G. Manara, and F. Gianne, "Location, Location, Location”, IEEE Vehicular Technology Magazine, Vol. 3; pp. 20-29, June 2008

[12] Bo-Chieh Liu; Ken-Huang Lin; SSSD-Based Mobile Positioning: On the Accuracy Improvement Issues in Distance and Location Estimations. IEEE Transactions on Vehicular Technology, Volume: 58, Issue: 3. 2009, Page(s): 1245 - 1254.

[13] Ouyang, R. W.; Wong, A. K.-S.; Lea, C.-T.; Received Signal Strength-Based Wireless Localization via Semidefinite Programming: Noncooperative and Cooperative Schemes. IEEE Transactions on Vehicular Technology, Volume: 59, Issue:3, 2010, pp: 1307 - 1318

[14] Parker, R.; Valaee, S.; Vehicular Node Localization Using Received-Signal-Strength Indicator. IEEE Transactions on Vehicular Technology, Volume: 56, Issue: 6, Part: 1 2007, pp: 3371 - 3380

[15] S.A.Mohamed, A. Nasr, G.A. Ansari, "Precise Positioning Systems for Vehicular Ad-hoc Networks using Received Signal Strength and Carbon Nanotube", submitted to Computer Journal, Oxford.

[16] Hossain, A.K.M.; Hien Nguyen Van; Yunye Jin; Wee-Seng Soh; "Indoor Localization Using Multiple Wireless Technologies", In IEEE Internatonal Conference on Mobile Adhoc and Sensor Systems, 2007. MASS 2007. pp: $1-8$.

[17] Rahman, M.Z.; Kleeman, L.; Paired Measurement Localization: A Robust Approach for Wireless Localization IEEE Transactions on Mobile Computing, Vol.: 8, Issue: 8, 2009, pp: 1087 - 1102

[18] R. Singh, M. Guainazzo, and C. S. Regazzoni, "Location determination using WLAN in conjunction with GPS network," in Proc. of the Vehicular Technology Conference (VTC-Spring), May 2004, pp. 2695-2699

[19] Bo-Chieh Liu; Lin, K.-H.; Jieh-Chian Wu; Analysis of hyperbolic and circular positioning algorithms using stationary signal-strength-difference measurements in wireless communications. IEEE Transactions on Vehicular Technology, Volume: 55, Issue: 2, 2006, pp: $499-509$.

[20] Mada, K.K.; Hsiao-Chun Wu; Iyengar, S.S.; Efficient and Robust EM Algorithm for Multiple Wideband Source Localization. IEEE Transactions on Vehicular Technology, Volume: 58, Issue: 6, 2009, pp: 3071 - 3075 
[21]X. Chen, Y. Chen, and F. Rao, "An Efficient Spatial Publish/Subscribe System for Intelligent Location-Based Services", Proceedings of the $2^{\text {nd }}$ International Workshop on Distributed EventBased Systems (DEBS'03), June 2003

[22] J. Hightower and G. Borriello, "Location Systems for Ubiquitous Computing," Computer, vol. 34, no. 8, pp. 57-66, IEEE Computer Society Press, August 2001

[23] L. Wischof, A. Ebner, and H. Rohling, "Information dissemination in self-organizing intervehicle networks", IEEE Trans. intelligent transportation systems, vol. 6, no. 1, pp. 90-101, Mar. 2005.

[24] Saad, S.S.; Nakad, Z.S.; , "A Standalone RFID Indoor Positioning System Using Passive Tags," IEEE Transactions on Industrial Electronics, vol.58, no.5, pp.1961-1970, May 2011 doi: 10.1109/TIE.2010.2055774

[25] Chen, Miao; Jin, Jishun; , "An Improvement Approach of Indoor Location Based on Radio-Map Using Wireless Local Area Network," 2011 International Conference on Computer and Management (CAMAN), pp.1-4, 19-21 May 2011, doi: 10.1109/CAMAN.2011.5778740

[26] Ming, Wang; Zhengqiu, Lu; Shan, Jin; Beng, Shao; Qingzhang, Chen; , "Design of an indoor positioning and tracking algorithm for wireless sensor networks," 2011 International Conference on Consumer Electronics, Communications and networks (CECNet), pp.3081-3084, 16-18 April 2011doi: 10.1109/CECNET.2011.5768290

[27] Shum, K.C.Y.; Quan Jia Cheng; Ng, J.K.Y.; Ng, D.; , "A Signal Strength Based Location Estimation Algorithm within a Wireless Network," 2011 IEEE International Conference on Advanced Information Networking and Applications (AINA), pp.509-516, 22-25 March 2011doi: 10.1109/AINA.2011.80

\section{Author}

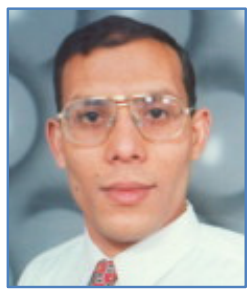

Samir A. Elsagheer Mohamed obtained a B.Sc. degree in Computer and Control Systems, from the Faculty of Engineering at the University of Assuit, Egypt, in May 1994. He worked as a teaching assistant in the Faculty of Engineering at Aswan from 1995 to 1997. He obtained his M.Sc. degree in Computer Science from the University of Rennes I, France, in 1998. He obtained his Ph.D. degree in Computer Engineering from the INRIA/IRISA, University of Rennes, France, in January 2003. Then, he worked as R\&D Expert Engineer at the INRIA/IRISA until June 2006. After that he moved to the Faculty of Engineering at Aswan (Egypt) to work as assistant professor. Currently, he is with the College of Computer, Computer Engineering Department, at the Qassim University, Saudi Arabia. He is also the manager of the research unit in the College of Computer. His research interests are in vehicular ad hoc networks, ad hoc network security, audio and video quality assessment in computer networks; rate based control mechanisms; video codecs; sensor networks; traffic prediction; learning algorithms for neural networks; text classifications; cryptography; and road traffic safety. 